\title{
Diophantine Equations in Partitions
}

\author{
By Hansraj Gupta
}

\begin{abstract}
Given positive integers $r_{1}, r_{2}, r_{3}, \ldots, r_{j}$ such that

$$
r_{1}<r_{2}<r_{3}<\cdots<r_{j}<m ; \quad m>1 ;
$$

we find the number $P(n, m ; R)$ of partitions of a given positive integer $n$ into parts belonging to the set $R$ of residue classes

$$
r_{1}(\bmod m), \quad r_{2}(\bmod m), \ldots, r_{j}(\bmod m) .
$$

This leads to an identity which is more general though less elegant then the well-known Rogers-Ramanujan identities.
\end{abstract}

1. Notation. In what follows, small letters other than $x$ denote nonnegative integers; $|x|<1$; the parts in a partition are deemed to be arranged in a nonascending order unless otherwise clear from the context; $p(u, v)$ denotes the number of partitions of $u$ into at most $v$ parts, with $p(0, v)=1$; square brackets denote the greatest integer function; $m>1$; and we write

$$
X\left(a_{i}\right) \text { for } 1 /\left\{(1-x)\left(1-x^{2}\right) \cdots\left(1-x^{a_{i}}\right)\right\} \quad \text { with } X(0)=1 \text {. }
$$

2. The Problem. Given $m>1$, and integers $r_{1}, r_{2}, r_{3}, \ldots, r_{j}$ such that

$$
0<r_{1}<r_{2}<\cdots<r_{j}<m \text {; }
$$

we have to find the number $P(n, m ; R)$ of partitions of a given number $n$ into parts belonging to the set $R$ of residue classes

$$
r_{1}(\bmod m), \quad r_{2}(\bmod m), \ldots, r_{j}(\bmod m)
$$

if any such exist.

Let us first consider the set of those partitions of $n$ which have

$$
\begin{aligned}
& a_{1} \text { parts each congruent to } r_{1}(\bmod m), \\
& a_{2} \text { parts each congruent to } r_{2}(\bmod m), \\
& a_{j} \text { parts each congruent to } r_{j}(\bmod m) .
\end{aligned}
$$

For $n$ to have such a partition, it is necessary that

$$
n=r_{1} a_{1}+r_{2} a_{2}+\cdots+r_{j} a_{j}+C m
$$

for some integer $C \geqslant 0$.

Any partition belonging to our set can be considered to have $j$ sections-the first consisting of $a_{1} r_{1}$ 's, the second of $a_{2} r_{2}$ 's; $\ldots$, and the $j$ th of $a_{j} r_{j}$ 's.

Received June 21, 1982.

1980 Mathematics Subject Classification. Primary 10A45, 10B05; Secondary 05A17. 
To get a partition of $n$ of the desired type, we must distribute the $C m$ 's between the $j$ sections in all possible ways. Let $c_{1} m$ 's be allotted to the first section, $c_{2}$ to the second section,.... and $c_{j}$ to the $j$ th section.

Since the elements of each section are all alike. the $c_{i} m$ 's assigned to the $i$ th section are partitioned into at most $a_{i}$ parts which are then tagged on to the elements of the section in order. Thus the number of partitions to which the allotment leads is given by

$$
p\left(c_{1}, a_{1}\right) \cdot p\left(c_{2}, a_{2}\right) \cdot p\left(c_{3}, a_{3}\right) \cdots p\left(c_{j}, a_{j}\right) .
$$

Letting $c_{1}, c_{2}, \ldots, c_{j}$ run through all the $\left(\begin{array}{c}c+j-1 \\ 1,1\end{array}\right)$ solutions of the Diophantine equation

$$
c_{1}+c_{2}+c_{3}+\cdots+c_{j}=C
$$

in nonnegative integers, we can not only find the number of partitions in our set but can also write them out. We do this in the following example with

$$
\begin{aligned}
& m=11 ; \quad r_{1}=2, r_{2}=6, r_{3}=8, r_{4}=10 ; \\
& a_{1}=5, \quad a_{2}=2, a_{3}=1, a_{4}=1 ; \quad \text { and } C=3 ;
\end{aligned}
$$

which implies that $n=73$.

The Diophantine equation

\begin{tabular}{|c|c|c|c|c|c|c|}
\hline & $c_{1}$ & $c_{2}$ & $c_{3}$ & $c_{4}$ & Partitions & Number \\
\hline 1. & 3 & 0 & 0 & 0 & $\begin{array}{l}35.2,2,2,2: 6,6: 8: 10: \\
24.13,2: 2: 2: 6: 6: 8: 10: \\
13.13 i 13,2: 2: 6,6: 8: 10:\end{array}$ & 3 \\
\hline 2. & 0 & 3 & 0 & 0 & 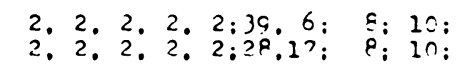 & 2 \\
\hline $\begin{array}{l}3 . \\
4 .\end{array}$ & $\begin{array}{l}0 \\
0 \\
2\end{array}$ & $\begin{array}{l}0 \\
0 \\
1\end{array}$ & $\begin{array}{l}3 \\
c \\
0\end{array}$ & $\begin{array}{l}0 \\
3 \\
0\end{array}$ & 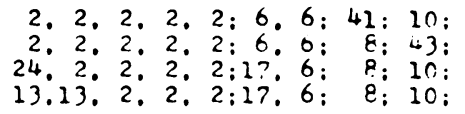 & $\begin{array}{l}1 \\
1 \\
2\end{array}$ \\
\hline 6. & 2 & 0 & 1 & 0 & $\begin{array}{l}24,2,2,2,2: 6,6: 19: 10: \\
13,13,2,2,2: 6,6: 19: 10:\end{array}$ & 2 \\
\hline 7. & 2 & 0 & 0 & 1 & $\begin{array}{l}24,2,2,2,2: 6,6: 8: 21 ; \\
13,13,2,2,2: 6,6: 8: 21:\end{array}$ & 2 \\
\hline 8. & 1 & 2 & 0 & 0 & $\begin{array}{lll}13,2,2,2,2: 28,6: & 8: 10: \\
13,2,2,2,2: 17,17: & 8: 10:\end{array}$ & 2 \\
\hline 9. & 0 & 2 & 1 & 0 & $\begin{array}{l}2,2,2,2: 29,6: 19: 10: \\
2,2,2,2,2: 17,17: 19: 10:\end{array}$ & 2 \\
\hline 10. & 0 & 2 & 0 & 1 & 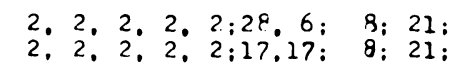 & 2 \\
\hline $\begin{array}{l}11 . \\
12 . \\
13 . \\
14 . \\
15 . \\
16 . \\
1 ? . \\
18 . \\
13 . \\
20 .\end{array}$ & $\begin{array}{l}0 \\
0 \\
1 \\
0 \\
0 \\
1 \\
1 \\
1 \\
1 \\
0\end{array}$ & $\begin{array}{l}0 \\
1 \\
0 \\
0 \\
1 \\
0 \\
1 \\
1 \\
1 \\
1\end{array}$ & $\begin{array}{l}2 \\
2 \\
2 \\
1 \\
0 \\
0 \\
1 \\
0 \\
1 \\
1\end{array}$ & $\begin{array}{l}1 \\
0 \\
0 \\
2 \\
2 \\
2 \\
i \\
1 \\
1 \\
1\end{array}$ & 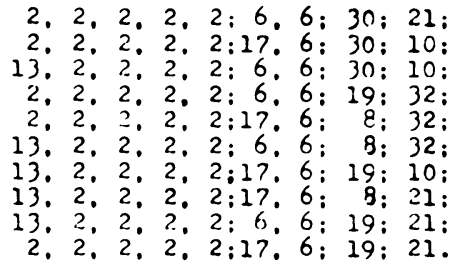 & $\begin{array}{l}1 \\
1 \\
1 \\
1 \\
1 \\
1 \\
1 \\
1 \\
1 \\
1\end{array}$ \\
\hline
\end{tabular}

$$
c_{1}+c_{2}+c_{3}+c_{4}=3
$$

has 20 solutions. We present them in the following table along with the partitions to which they give rise and their number. 
Thus the required number of partitions in the set is 29. A formula for the number of partitions in the set is obtained as follows. We note that $p\left(c_{i}, a_{i}\right)$ is the coefficient of $x^{c}$ in the expansioin of $X\left(a_{i}\right)$. Hence

$$
\sum_{c} p\left(c_{1}, a_{1}\right) \cdot p\left(c_{2}, a_{2}\right) \cdots \cdots p\left(c_{j}, a_{j}\right)
$$

where $c$ 's run over all the solutions of the Diophantine equation (3), is the coefficient of $x^{(}$in the expansion (in ascending powers of $x$ ) of the product

$$
X\left(a_{1}\right) \cdot X\left(a_{2}\right) \cdot X\left(a_{3}\right) \cdots X\left(a_{j}\right) .
$$

This is the same as the coefficient of $x^{C}$ in

$$
\begin{gathered}
X\left(b_{1}\right) \cdot X\left(b_{2}\right) \cdot X\left(b_{3}\right) \cdots X X\left(b_{j}\right), \text { where } \\
b_{i}=\min \left(C, a_{i}\right), \quad i=1,2,3, \ldots, j .
\end{gathered}
$$

In our example, it is the coefficient of $x^{3}$ in

$$
X(3) \cdot X(2) \cdot X(1) \cdot X(1) \text {. }
$$

We leave it to the reader to verify that the coefficient is 29 .

3. The Formula for $P(n, m ; R)$. We have seen that

$$
r_{1} a_{1}+r_{2} a_{2}+r_{3} a_{3}+\cdots+r_{j} a_{j}=n-C m \text {. }
$$

In this let $C$ take in succession the values $0,1,2, \ldots,[n / m]$. For each of these values, regarding (6) as a Diophantine equation in $a$ 's, find all the solutions of (6) and the contribution of each such solution to $P(n, m ; R)$. Then we get

$$
\begin{aligned}
& P(n, m ; R)=\text { the sum of these contributions; } \\
& =\sum_{C=0}^{[n / m]} \text { coefficient of } x^{C} \text { in } X\left(a_{1}\right) X\left(a_{2}\right) \cdots X\left(a_{j}\right),
\end{aligned}
$$

where each $a_{i}>C$ can be replaced by $C$.

The following examples will show how the calculations can best be presented.

Example 1. Let $m=5, r_{1}=2, r_{2}=3$ and $n=25$.

Our presentation will be as follows:

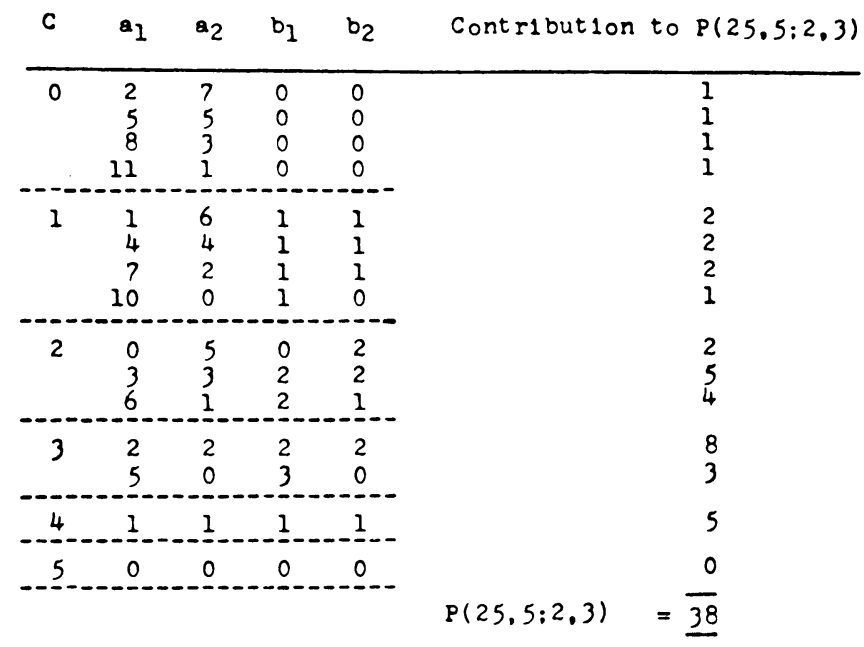


By the second Rogers-Ramanujan identity, we will have

$$
\begin{aligned}
& P(25,5 ; 2,3)=p(23,1)+p(19,2)+p(13,3)+p(5,4) \text {, } \\
& =1+10+21+6=38 \text {. }
\end{aligned}
$$

Before we consider our next example, let it be recalled that the number of solutions of (6) is the coefficient of $x^{n-C m}$ in

$$
\left\{\left(1-x^{r_{1}}\right)\left(1-x^{r_{2}}\right)\left(1-x^{r_{3}}\right) \cdots\left(1-x^{r_{1}}\right)\right\}^{-1} \text {. }
$$

We leave it to the reader to verify this in the above example.

Example 2. Let $m=7 ; r=1, r=2, r=4 ; n=25$. In this case (8) gives the following information:

$\begin{array}{crrrr}C: & 0 & 1 & 2 & 3 \\ \text { Number of solutions: } & 49 & 30 & 12 & 4 .\end{array}$

Our calculations are a little more elaborate this time.

\begin{tabular}{cccccccc}
$c$ & $a_{1}$ & $a_{2}$ & $a_{3}$ & $b_{1}$ & $b_{2}$ & $b_{3}$ & $p$ \\
\hline 0 & 1 & 0 & 6 & 0 & 0 & 0 & 1 \\
& 5 & 0 & 5 & 0 & 0 & 0 & 1 \\
& 3 & 1 & 5 & 0 & 0 & 0 & 1 \\
1 & 2 & 5 & 0 & 0 & 0 & 1 \\
9 & 0 & 4 & 0 & 0 & 0 & 1 \\
7 & 1 & 4 & 0 & 0 & 0 & 1 \\
& 5 & 2 & 4 & 0 & 0 & 0 & 1 \\
& 3 & 3 & 4 & 0 & 0 & 0 & 1 \\
1 & 4 & 4 & 0 & 0 & 0 & 1 \\
& 13 & 0 & 3 & 0 & 0 & 0 & 1
\end{tabular}

\begin{tabular}{cccccccc}
$c$ & $a_{1}$ & $a_{2}$ & $a_{3}$ & $b_{1}$ & $b_{2}$ & $b_{3}$ & $p$ \\
\hline 1 & 2 & 0 & 4 & 1 & 0 & 1 & 2 \\
& 0 & 1 & 4 & 0 & 1 & 1 & 2 \\
& 6 & 0 & 3 & 1 & 0 & 1 & 2 \\
& 4 & 1 & 3 & 1 & 1 & 1 & 3 \\
2 & 2 & 3 & 1 & 1 & 1 & 3 \\
0 & 3 & 3 & 0 & 1 & 1 & 2 \\
& 10 & 0 & 2 & 1 & 0 & 1 & 2 \\
8 & 1 & 2 & 1 & 1 & 1 & 3 \\
& 6 & 2 & 2 & 1 & 1 & 1 & 3 \\
4 & 3 & 2 & 1 & 1 & 1 & 3
\end{tabular}

$\begin{array}{rrrrrrr}11 & 1 & 3 & 0 & 0 & 0 & 1 \\ 9 & 2 & 3 & 0 & 0 & 0 & 1 \\ 7 & 3 & 3 & 0 & 0 & 0 & 1 \\ 5 & 4 & 3 & 0 & 0 & 0 & 1 \\ 3 & 5 & 3 & 0 & 0 & 0 & 1 \\ 1 & 6 & 3 & 0 & 0 & 0 & 1 \\ 17 & 0 & 2 & 0 & 0 & 0 & 1 \\ 15 & 1 & 2 & 0 & 0 & 0 & 1 \\ 13 & 2 & 2 & 0 & 0 & 0 & 1 \\ 11 & 3 & 2 & 0 & 0 & 0 & 1\end{array}$

$\begin{array}{rrrrrrr}2 & 4 & 2 & 1 & 1 & 1 & 3 \\ 0 & 5 & 2 & 0 & 1 & 1 & 2 \\ 14 & 0 & 1 & 1 & 0 & 1 & 2 \\ 12 & 1 & 1 & 1 & 1 & 1 & 3 \\ 10 & 2 & 1 & 1 & 1 & 1 & 3 \\ 8 & 3 & 1 & 1 & 1 & 1 & 3 \\ 6 & 4 & 1 & 1 & 1 & 1 & 3 \\ 4 & 5 & 1 & 1 & 1 & 1 & 3 \\ 2 & 6 & 1 & 1 & 1 & 1 & 3 \\ 0 & 7 & 1 & 0 & 1 & 1 & 2\end{array}$

$\begin{array}{lllllll}9 & 4 & 2 & 0 & 0 & 0 & 1 \\ 7 & 5 & 2 & 0 & 0 & 0 & 1 \\ 5 & 6 & 2 & 0 & 0 & 0 & 1 \\ 3 & 7 & 2 & 0 & 0 & 0 & 1 \\ 1 & 8 & 2 & 0 & 0 & 0 & 1 \\ 21 & 0 & 1 & 0 & 0 & 0 & 1 \\ 19 & 1 & 1 & 0 & 0 & 0 & 1 \\ 17 & 2 & 1 & 0 & 0 & 0 & 1 \\ 15 & 3 & 1 & 0 & 0 & 0 & 1 \\ 13 & 4 & 1 & 0 & 0 & 0 & 1\end{array}$

$\begin{array}{rllllll}18 & 0 & 0 & 1 & 0 & 0 & 1 \\ 16 & 1 & 0 & 1 & 1 & 0 & 2 \\ 14 & 2 & 0 & 1 & 1 & 0 & 2 \\ 12 & 3 & 0 & 1 & 1 & 0 & 2 \\ 10 & 4 & 0 & 1 & 1 & 0 & 2 \\ 8 & 5 & 0 & 1 & 1 & 0 & 2 \\ 6 & 6 & 0 & 1 & 1 & 0 & 2 \\ 4 & 7 & 0 & 1 & 1 & 0 & 2 \\ 2 & 8 & 0 & 1 & 1 & 0 & 2 \\ 0 & 9 & 0 & 0 & 1 & 0 & 1\end{array}$




\begin{tabular}{|c|c|c|c|c|c|c|c|c|c|c|c|c|c|c|}
\hline$a_{1}$ & $a_{2}$ & $a_{3}$ & $b_{1}$ & $\mathrm{~b}_{2}$ & $b_{3}$ & $\mathbf{P}$ & C & $a_{1}$ & h & $a_{3}$ & $b_{1}$ & $b_{2}$ & D & $P$ \\
\hline $\begin{array}{r}11 \\
9 \\
7 \\
5 \\
3 \\
1 \\
25 \\
23 \\
21 \\
19\end{array}$ & $\begin{array}{r}5 \\
6 \\
7 \\
8 \\
9 \\
10 \\
0 \\
1 \\
2 \\
3\end{array}$ & $\begin{array}{l}1 \\
1 \\
1 \\
1 \\
1 \\
1 \\
0 \\
0 \\
0 \\
0\end{array}$ & $\begin{array}{l}0 \\
0 \\
0 \\
0 \\
0 \\
0 \\
0 \\
0 \\
0 \\
0\end{array}$ & $\begin{array}{l}0 \\
0 \\
0 \\
0 \\
0 \\
0 \\
0 \\
0 \\
0 \\
0\end{array}$ & $\begin{array}{l}0 \\
0 \\
0 \\
0 \\
0 \\
0 \\
0 \\
0 \\
0 \\
0\end{array}$ & $\begin{array}{l}1 \\
1 \\
1 \\
1 \\
1 \\
1 \\
1 \\
1 \\
1 \\
1\end{array}$ & 2 & $\begin{array}{r}3 \\
1 \\
7 \\
5 \\
3 \\
1 \\
11 \\
9 \\
7 \\
5\end{array}$ & $\begin{array}{l}0 \\
1 \\
0 \\
1 \\
2 \\
3 \\
0 \\
1 \\
2 \\
3\end{array}$ & $\begin{array}{l}2 \\
2 \\
1 \\
1 \\
1 \\
1 \\
0 \\
0 \\
0 \\
0\end{array}$ & $\begin{array}{l}2 \\
1 \\
2 \\
2 \\
2 \\
1 \\
2 \\
2 \\
2 \\
2\end{array}$ & $\begin{array}{l}0 \\
1 \\
0 \\
1 \\
2 \\
2 \\
0 \\
1 \\
2 \\
2\end{array}$ & $\begin{array}{l}2 \\
2 \\
1 \\
1 \\
1 \\
1 \\
0 \\
0 \\
0 \\
0\end{array}$ & $\begin{array}{l}5 \\
7 \\
4 \\
7 \\
8 \\
7 \\
2 \\
4 \\
5 \\
5\end{array}$ \\
\hline $\begin{array}{l}17 \\
15 \\
13\end{array}$ & $\begin{array}{l}4 \\
5 \\
6\end{array}$ & $\begin{array}{l}0 \\
0 \\
0\end{array}$ & $\begin{array}{l}0 \\
0 \\
0\end{array}$ & $\begin{array}{l}0 \\
0 \\
0\end{array}$ & $\begin{array}{l}0 \\
0 \\
0\end{array}$ & $\begin{array}{l}1 \\
1 \\
1\end{array}$ & & $\begin{array}{l}3 \\
1\end{array}$ & $\begin{array}{l}4 \\
5\end{array}$ & $\begin{array}{l}0 \\
0\end{array}$ & $\begin{array}{l}2 \\
1\end{array}$ & $\begin{array}{l}2 \\
2 .\end{array}$ & $\begin{array}{l}0 \\
0\end{array}$ & 5 \\
\hline $\begin{array}{r}17 \\
9 \\
7 \\
5\end{array}$ & $\begin{array}{r}? \\
8 \\
9 \\
10\end{array}$ & $\begin{array}{l}0 \\
0 \\
0 \\
0\end{array}$ & $\begin{array}{l}0 \\
0 \\
0 \\
0\end{array}$ & $\begin{array}{l}0 \\
0 \\
0 \\
0\end{array}$ & $\begin{array}{l}0 \\
0 \\
0 \\
0\end{array}$ & $\begin{array}{l}1 \\
1 \\
1 \\
1 \\
1\end{array}$ & 3 & $\begin{array}{l}0 \\
4 \\
2 \\
0\end{array}$ & $\begin{array}{l}0 \\
0 \\
1 \\
2\end{array}$ & $\begin{array}{l}1 \\
0 \\
0 \\
0\end{array}$ & $\begin{array}{l}0 \\
3 \\
2 \\
0\end{array}$ & $\begin{array}{l}0 \\
0 \\
1 \\
2\end{array}$ & $\begin{array}{l}1 \\
0 \\
0 \\
0\end{array}$ & $\begin{array}{l}1 \\
3 \\
6 \\
2\end{array}$ \\
\hline 3 & & 0 & 0 & 0 & 0 & & & & & & & & & \\
\hline
\end{tabular}

To check our result, we make use of the well-known fact that $P(n, m ; R)$ is the coefficient of $x^{n}$ in the expansion of

$$
\prod_{q=0}^{[n / m]}\left\{\prod_{i=1}^{j}\left(1-x^{r_{i}+q m}\right)\right\}^{-1} \text {. }
$$

In our example (9) is

$$
\begin{aligned}
\left\{(1-x)\left(1-x^{2}\right)\left(1-x^{4}\right) \cdot\left(1-x^{8}\right)\left(1-x^{9}\right)\left(1-x^{11}\right)\right. \\
\left.\cdot\left(1-x^{15}\right)\left(1-x^{16}\right)\left(1-x^{18}\right) \cdot\left(1-x^{22}\right)\left(1-x^{23}\right)\left(1-x^{25}\right)\right\}^{-1} .
\end{aligned}
$$

Expanding this, we obtained the following table of coefficients of $x^{n}, 0 \leqslant n \leqslant 25$.

\begin{tabular}{rrrrrrrrrrr} 
n & 0 & 1 & 2 & 3 & 4 & 5 & 6 & 7 & 8 & 9 \\
\hline 0 & 1 & 1 & 2 & 2 & 4 & 4 & 6 & 6 & 10 & 11 \\
1 & 15 & 17 & 23 & 26 & 32 & 37 & 47 & 54 & 66 & 76 \\
2 & 93 & 105 & 126 & 143 & 172 & 194 & & & & \\
\hline
\end{tabular}

Incidentally, equating the results in (7) and (9), we get an identity which is more general but not as elegant as the well-known Rogers-Ramanujan identities.

Department of Mathematics

Panjab University

Chandigarh 160014, India 